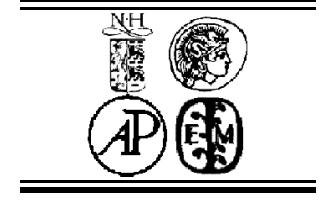

\title{
Hysteretic behavior of spin-crossover noise driven system
}

\author{
Iurii Gudyma $^{\mathrm{a}}$, Artur Maksymov ${ }^{\mathrm{a}, \mathrm{b}^{*}}$, Mihai Dimian ${ }^{\mathrm{c}, \mathrm{d}}$ \\ ${ }^{a}$ Department of General Physics, Chernivtsi National University, Chernivtsi 58012, Ukraine. \\ ${ }^{\mathrm{b}}$ Advanced Material Research Institute, University of New Orleans, LA 70148, USA. \\ ${ }^{\mathrm{c}}$ Department of Electrical and Computer Engineering, Howard University, Washington, DC 20059, USA. \\ ${ }^{c}$ Faculty of Electrical Engineering and Computer Science, Stefan cel Mare University, Suceava, 720229, Romania.
} Elsevier use only: Received date here; revised date here; accepted date here

\begin{abstract}
The influence of white Gaussian noise on hysteretic behavior of spin-crossover system is analyzed in the framework of stochastic Langevin dynamics. Various stochastic simulations are performed and several important properties of spintransition in spin-crossover system driven by noise are reproduced. The numerical results are tested against the stationary probability function and the associated dynamic potential obtained from Fokker-Planck equation corresponding to spincrossover Langevin dynamics. The dependence of light-induced optical hysteresis width and non-hysteretic transition curve slope on the noise intensity is illustrated. The role of low-spin and high-spin phase stabilities in the hysteretic behavior of noise-driven spin-crossover system is discussed. () 2015 Elsevier Science. All rights reserved
\end{abstract}

Keywords: light-induced spin transition, spin-cossover, noise.

\section{Introduction}

The recent researches for new materials able to satisfy the increasing requirements in information storage technology have shown that spin-crossover compounds are promising candidates for developing novel data storage devices. The spin-crossover compounds are coordination inorganic complexes with $d^{4}-d^{7}$ electronic configuration of metal ion orbitals, situated in the center of the octahedral ligand field [1-3]. The main property, which makes these molecular magnetic materials attractive to data storage is bistability controlled by external physical fields. This bistable property is related to the existence of low-spin (LS) and high-spin (HS) configuration of degenerated $d$-orbitals of metal ion, which takes places due to the action of crystal field of the surrounded ligand and lead to the existence of hysteresis loop controlled by external field [4-6].

In the most cases the spin-crossover system is fully described by the behavior of fraction of high-spin molecules, which depends on the action of physical fields (magnetic, light irradiation, temperature, pressure and other). In this work we focused on the hysteretic properties of the spin-crossover systems induced by light irradiation ( $\mathrm{LIOH}$ - light induced optical hysteresis) in presence of noise, describing the fluctuations of system parameters. The theoretical framework for studying the stochastic behavior of spin-crossover systems was developed in [7, 8] by describing the interaction with environment as contact with a heat bath [9] and by using the stochastic Langevin dynamics in connection to a set of Fokker-Plank equations.

\section{Theoretical model}

For studying the hysteretic behavior of a spincrossover compound let us start from the phenomenological model of high-spin fraction evolution and consider the reciprocal concurrence between relaxation and photoexcitation processes, which accounts for the decreasing or the increasing of molecular magnetic moment. The resulting dynamics of the system can be described by the following kinetic equation $[10,11]$ :

$\frac{d n_{H}}{d t}=f_{e x c}\left(n_{H}, t\right)-f_{r e l}\left(n_{H}, t\right) \equiv f\left(n_{H}, t\right)$

where excitation and relaxation processes are termed as follows:

$$
\begin{aligned}
& f_{\text {exc }}\left(n_{H}, t\right)=\beta\left(1-n_{H}\right), \\
& f_{\text {rel }}\left(n_{H}, t\right)=-n_{H} \exp \left(-\alpha n_{H}\right) .
\end{aligned}
$$

Here, the coefficient $\beta$ in excitation term accounts

“Corresponding author: E-mail address: maxyartur@gmail.com (Artur Maksymov) 
for the effects of light absorption during the irradiation of spin-crossover sample by the specific cross-section, leading to the change of spin configuration from LS to the HS one. The physical meaning of this coefficient is the transition probability per time unit from ground LS to the metastable HS state, induced by external control field [9]. In the same time, the rate of relaxation is characterized by self-acceleration factor $\alpha$, which is defined by cooperative effects in the system.

A more rigorous exploration of the system dynamics, especially on finite time interval, requires the consideration of non-stationary terms arisen from the different kind of external and internal irregularities, which introduce in the system a certain degree of disorder. The theoretical description of such system dynamics may be provided by assuming the presence of any random forces described as background noise actions. The simplest description of this stochastic dynamics assumes that the background random forces are independent on system variables and uncorrelated between themselves. This implies the Langevin kinetic description with additive noise action of the following form:

$$
\frac{d n_{H}}{d t}=f_{e x c}\left(n_{H}, t\right)-f_{r e l}\left(n_{H}, t\right)+\xi(t)
$$

where the stochastic processes $\xi(t)$ describes additive noise action and is characterized by Gaussian distributions with the following statistical properties:

$$
\begin{aligned}
& \langle\xi(t)\rangle=0 \\
& \left\langle\xi(t) \xi\left(t^{\prime}\right)\right\rangle=2 \varepsilon^{2} \delta\left(t-t^{\prime}\right)
\end{aligned}
$$

The parameter $\varepsilon^{2}$ from correlation function specifies the intensity of additive white Gaussian noise.

The statistical description of system behavior in terms of the evolution of the probability distribution function $P\left(n_{s}, t\right)$ is provided by the Fokker-Planck equation associated to Langevin kinetic equation (3). For the system with additive noise, the FokkerPlanck equation reads as follows:

$$
\frac{\partial P\left(n_{H}, t\right)}{\partial t}=-\frac{\partial}{\partial n_{H}} f\left(n_{H}, t\right) P\left(n_{H}, t\right)+\varepsilon \frac{\partial^{2}}{\partial t^{2}} P\left(n_{H}, t\right)(5)
$$

The possibility to characterize the evolution of probability density function of the system in the Fokker-Planck formalism gives the opportunity to estimate the system stability and may be applied to dynamical system by introducing the dynamic potential in terms of Lyapunov function. In this case, the stationary solution of Fokker-Planck equation (5) represents the probability in the Gibbs-Boltzmann form
$P_{s t}\left(n_{H}\right)=N \exp \left(-U\left(n_{H}\right)\right)$

with a normalization constant $N$ and the following dynamic potential

$U\left(n_{H}\right)=-\int_{0}^{n_{H}} f(x) / \varepsilon^{2} d x$.

A more detailed discussion of Fokker-Planck method to similar problem is given in $[7,9,12,13]$.

The analytical expression of light-induced optical hysteresis $(\mathrm{LIOH})$ for stationary dynamics can be easily found from Eq. (1) by considering Eq. (2) and time $t \rightarrow \infty$ [13]. The situation is significantly different for finite time and the presence of noise, where no exact analytical solution is available and the analysis of stochastic behavior is possible by numerical simulations.

For the system dynamics described by Eq. (3), and taking into account Eqs. (2) and (4), the numerical solution can be found by Heune algorithm based on second-order Runge-Kutta type methods, and has the following general description [14-16]:

$$
\begin{aligned}
& y(t)=n_{H}(t)+h f\left(n_{H}, t\right)+\sqrt{h} \varepsilon^{2} u(t) ; \\
& n_{H}(t+h)=n_{H}(t)+\frac{h}{2}\left[f\left(n_{H}, t\right)+f(y, t+h)\right]+\frac{\sqrt{h}}{2} \varepsilon^{2} u(t) .
\end{aligned}
$$

Here $h$ is integration time step and $u(t)$ is a Gaussian random variable with zero mean value and variance equal to 1 . The dynamic potentials can be also found as the logarithm of probability distribution functions (based on Eq. (6)), which is obtained from the ensemble of simulated trajectories. In order to generate this latter histogram, the range of possible values for HS fraction $n_{H}$ was divided into small regions and the number of solutions from the entire ensemble belonging to the each region was counted. The final numerical probability distribution was normalized based on the condition $\int_{-\infty}^{\infty} P\left(n_{H}\right) d n_{H}=1$.

\section{Result and Discussion}

The system parameters in the kinetic equations can be chosen to describe a wide class of iron(II) based spin-crossover compounds. In this paper, we focused on $\left[\mathrm{Fe}_{\mathrm{x}} \mathrm{Co}_{\mathrm{y}}(\mathrm{btr})(\mathrm{NCS})_{2}\right] \cdot \mathrm{H}_{2} \mathrm{O}$ complex which features light induced bistability and is characterized by self-acceleration factor $\alpha=5.14$ (the transition temperature of this spin-crossover complexes is at $T=53 \mathrm{~K}$ ). In numerical simulations we consider an ensemble of 300 independent trajectories with 100000 time steps each. Each point of averaged 
trajectory over chosen ensemble represents the system position in the phase space at corresponding time step.

The dependence of HS fraction $n_{H}$ on light intensity was obtained in two steps: firstly it was generated the averaged trajectory for each value of $\beta$, and then, there was obtained the average value of HS fraction $n_{H}$ over last 10000 time steps. The time average over last 10000 steps, which corresponds to $10 \%$ of entire simulated time, was chosen because of the considerations that the system reaches its steady solution. Following this sequence, the dependence of light induced hysteresis width on additive noise intensity was explored and the results are shown in Fig. 1. From this figure it is clearly seen that the presence of noise decreases the hysteresis width of the system, which is in agreement with the results previously observed for temperature hysteresis of spin-crossover system with fluctuations [17]. In the Fig. 1 are presented the transition curves for the firstorder phase transition regimes of the system for noiseless case marked by square (black online) and for several fixed noise values: $\varepsilon=0.005$ marked by circles (blue online), $\varepsilon=0.01$ marked by triangles up (pink online), $\varepsilon=0.015$ marked by triangles down (dark yellow online).

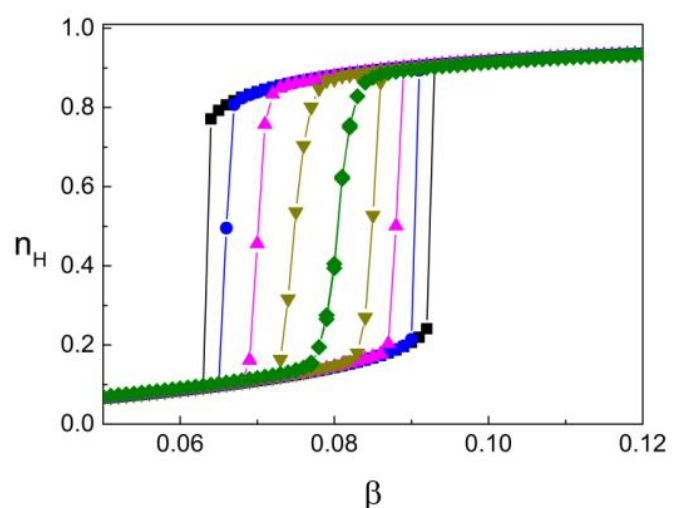

Fig 1. The light induced optical hysteresis, (LIOH) in the phase transition regime.

As displayed in Fig.1, the increase of noise intensity narrows the light induced hysteresis loop with nonlinear character up to its disappearance at noise value $\varepsilon=0.025$ which is the threshold one between hysteretic and nonhysteretic behavior of the system. This curve marked by diamonds (green online) illustrates the light induced second-order phase transition from LS to HS states. As it is known, the hysteresis represents the time-based dependence of a system's output on present and past inputs. The dependence arises because the history affects the value of an internal state. To predict its future outputs, either its internal state or its history must be known. However the noise destroys this relationship and makes the formation of the hysteresis loop impossible.
The further increase of noise alters the slope of nonhysteretic transition curves, as shown in Fig. 2. This reflects the fact that the noise disrupts the order in the system. Here the curves marked by squares (blue online) corresponds to the noise intensity $\varepsilon=0.03$, the ones marked by circles (pink online) are for $\varepsilon=0.04$ and the curves with triangles up (dark yellow online) are for $\varepsilon=0.05$. Noteworthy is the position of intersection points for displayed curves which is shifted relative to the point of system equilibrium at $n_{H}=0.5$. Obviously, this shift is related to the difference in saturation of LS and HS states. As apparent from the Fig.2, the HS state becomes saturated faster than LS one, although for the larger noise intensity, the system saturation is less pronounced for the both states.

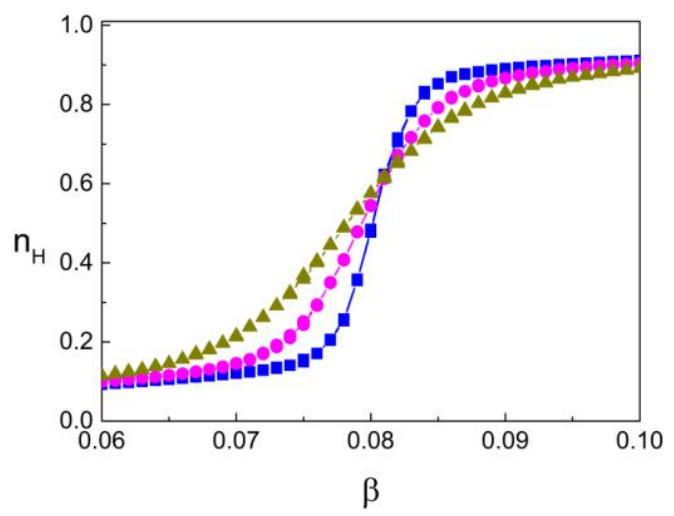

Fig 2. The simulated transition curves of spin-crossover system under noise in non-cooperative region.

To enhance the study of bistable behavior of the spin-crossover system under noise action it is interesting to find out the impact of noise influence on the dynamic potential. Fig.3 presents selected results, which is obtained for the stationary probability distributions of the system states from the Fokker-Plank equation (5) and the associated dynamic potentials. The analytical stationary probability (dashed line) and the associated dynamic potential (solid line) are compared to the ones obtained from the numerical simulation of Langevin equation (3), that was carried out according to the algorithm (8), for the ensemble of 300 trajectories (squared line) at the fixed noise intensities between $\varepsilon=0.015$ and $\varepsilon=0.05$ as indicated in the captions of the Fig.3. In order to understand better the system evolution on a finite time, the stochastic realizations of the system with initial conditions corresponding to LS and HS states are plotted on the right-hand side of Fig.3.

For relatively weak noise, the system location in LS or HS states is mainly defined by its initial conditions due to the relatively high potential barrier, so the transitions between the states are very rare events. The differences between analytical and numerical results observed in this case are simply 
related to the standard limitation of 10000 times steps imposed when computing the average value for $n_{H}$. These minor errors are acceptable for the purpose of computational efficiency of the general algorithm.

With increasing noise intensity, the height of dynamic potential decreases and the reciprocal transitions between the system states takes place, as reflected in Fig. 3 for $\varepsilon=0.025$. In this case, the dependence on the initial conditions is not maintained anymore and the trajectories which start from LS and HS states become synchronized in relatively short time.

The further increase of the noise intensity decreases the height of potential barrier and consequently, increases the number of transition per time unit, as it is reflected by the case $\varepsilon=0.05$ in Fig. 3.

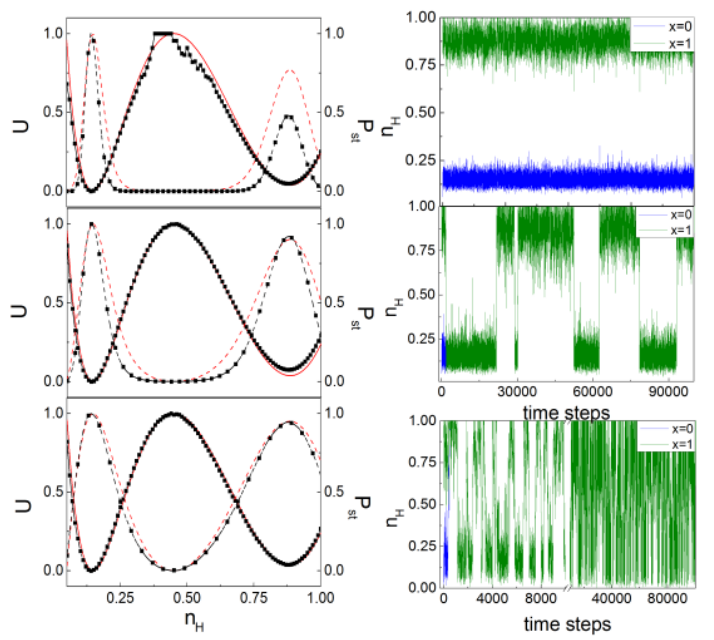

Fig 3. The analytical (solid [red online] line) and numerical (black squared line) normalized dynamic potential and corresponding probabilities of system states for the noise intensities (from top to bottom) $\varepsilon=0.015 \varepsilon=0.025$ and $\varepsilon=0.05$. On the right are displayed the dynamics of particular numerical stochastic trajectories for respective noise intensity.

By comparing the transition curves from Figs. 1 and 2 with the potentials which are shown in Fig. 3 one can understand better the effect of noise on the hysteretic behavior of the system. As it may be observed the width of hysteresis loop becomes zero at threshold noise intensity $\varepsilon=0.025$ for which is observed the bistable dynamical potential. Moreover, in the examined case with additive noise actions, for fixed value of control parameter $\beta$, the double well potential is maintained all the time indifferent to the value of noise intensity. Such kind of behavior may be explained by the same finite lifetime of HS and LS configurations. Furthermore, the noise action at the threshold intensity breaks the stability of the system states. For chosen intensity of light irradiation $\beta=0.081$, corresponding to the same depth of potential wells of LS and HS states, the escape from both LS and HS states may be observed at the threshold noise intensity or higher. Moreover, above the threshold value of additive noise, the favorable conditions for inter-state transitions take place, and they become very frequent. This is clearly seen from the behavior of trajectories shown in Fig. 3 on the right side, for the threshold noise and greater, and explains the smooth transition which is observed in Fig. 2.

\section{Conclusion}

In this paper, the noise driven dynamics of light induced spin-crossover system was examined in the framework of phenomenological kinetic equation. For the system with additive noise action, the behavior of light induced optical hysteresis (LIOH) was studied by numerical means. The stability of spin-crossover system was characterized by the dynamic potential derived from Fokker-Planck equations corresponding to the kinetic equation of evolution of high-spin fraction. It is find out that the hysteretic behavior of spin-crossover system depends on the stability of its LS and HS phases. When these phases are characterized by finite lifetime the reciprocal transition between the system states take place and the width of hysteresis loop becomes zero.

\section{Acknowledgments}

The work at University of New Orleans was supported by the National Science Foundation under the NSF EPSCoR Cooperative Agreement No. EPS1003897 with additional support from the Louisiana Board of Regents. The work at Suceava was partially supported by joint French-Romanian project ANRUEFISCDI, Contract No. 9RO-FR/ 01.02.2013. The authors acknowledge financial support for infrastructure development from the project "Integrated Center for research, development and innovation in Advanced Materials, Nanotechnologies, and Distributed Systems for fabrication and control", Contract No. 671/09.04.2015, POSCCE co-funded from the European Regional Development Fund.

\section{References}

[1] P. Gütlich, H. Goodwin (eds.), Spin Crossover in Transition Metal Compounds I-III, Springer, Berlin, 2004.

[2] M. A. Halcrow, Spin-Crossover Materials: Properties and Applications, John Wiley \& Sons, Chichester, 2013.

[3] Iu. Gudyma, C. Enachescu, A. Maksymov Springer Proceedings in Physics 156 (2015) 375.

[4] C. Enachescu, R. Tanasa, A. Stancu, G. Chastanet, J.-F. Létard, J. Linares, F. Varret, J. Appl. Phys. 99 (2006) 08J504.

[5] A. Atitoaie, R. Tanasa, A. Stancu, C. Enachescu, J. Magn. Magn. Mater. 368 (2014) 12.

[6] L. Stoleriu, A. Stancu, P. Chakraborty, A. Hauser, C. Enachescu, J. Appl. Phys. 117 (2015) 17 B307.

[7] Iu. Gudyma, A. Maksymov, S. Miyashita, Phys. Rev. E 84 (2011) 031126. 
[8] Iu. Gudyma, A. Maksymov, M. Dimian, Phys. Rev. E 88, (2013) 042111.

[9] Yu. Gudyma, O. Semenko, Phys. Stat. Sol. B 241 (2004) 370.

[10] A. Desaix, O. Roubeau, J. Jeftic, J.G. Haasnoot, K Boukheddaden, E. Codjovi, J. Linarès, M. Noguès, F. Varret, Eur. Phys. J. B 6 (1998) 183.

[11] Iu. V. Gudyma, A. Iu. Maksymov, Proc. of SPIE 9066 (2013) 906613

[12] Iu. Gudyma, A. Maksymov, C. Enachescu, Eur. Phys. J. B 78 (2010) 167.
[13] H. Risken, The Fokker-Planck Equation: Methods of Solution and Applications, Springer-Verlag, Berlin, 1989.

[14] M. San Miguel, R. Toral, in Instabilities and Nonequilibrium Structures VI, Dordrecht, Kluwer Academic Publishers, 2000, P. 35-130.

[15] Iu. V. Gudyma, A. Iu. Maksymov, Physica B 405 (2010) 2534.

[16] Iu. V. Gudyma, A. Iu. Maksymov, J. Phys. Chem. Solids 72 (2011) 73 .

[17] Iu. Gudyma, A. Maksymov, C. Enachescu, Phys. Rev. B 89 (2014) 224412. 